\title{
Corrosion Resistance and Passivation Behavior of B-containing S31254 Stainless Steel in a low pH medium
}

\author{
Muhammad Saqlain Qurashi ${ }^{1}$, Yishi Cui ${ }^{1}$, Jian Wang ${ }^{1, *}$, Nan Dong $^{1}$, Jingang Bai ${ }^{1,2}$, \\ Yucheng $\mathrm{Wu}^{1}$,Peide Han ${ }^{1, *}$ \\ ${ }^{1}$ College of Materials Science and Engineering, Taiyuan University of Technology, Taiyuan 030024, \\ China; \\ ${ }^{2}$ State Key Laboratory of Advanced Stainless Steel Materials, Taiyuan 030003, China; \\ *E-mail: jianwang1@126.com, hanpeide@126.com
}

doi: $10.20964 / 2019.11 .40$

Received: 14 June 2019 / Accepted: 21 August 2019 / Published: 7 October 2019

\begin{abstract}
Addition of boron is found to be effective in achieving corrosion resistance and passivation film composition of S31254 stainless steels in a harsh medium that has a low pH. Successfully enhanced corrosion resistance in chloride solutions with different $\mathrm{pH}$ and hindrance of the generation of donor densities with semiconducting characteristics are proved by potentiodynamic polarization tests, electrochemical impedance spectroscopy and Mott-Schottky analysis of different parameters. After potentiodynamic polarization, optical microscopic and scanning electron microscopy of the microstructure were analyzed. Results indicate that borated samples become free from pits that serve as initiation sites around the grain boundaries. Passive film characterization using XPS peak deconvoluted spectra and cationic fractions reveal that after boron addition primary elements of the inner and outer passive layers changed significantly. These changes enhanced the compactness, strength and repassivating ability of the film, which is further supported by XPS depth profile studies with respect to atomic and cationic concentrations.
\end{abstract}

Keywords: Super austenitic Stainless steel; Boron effect; Corrosion; passive film

\section{$\underline{\text { FULL TEXT }}$}

(C) 2019 The Authors. Published by ESG (www.electrochemsci.org). This article is an open access article distributed under the terms and conditions of the Creative Commons Attribution license (http://creativecommons.org/licenses/by/4.0/). 